\title{
Dynamic Offline Conflict-Free Coloring for Unit Disks
}

\author{
Joseph Wun-Tat Chan ${ }^{1}$, Francis Y.L. Chin ${ }^{2, \star}$, Xiangyu Hong ${ }^{3}$, \\ and Hing Fung Ting ${ }^{2, \star \star}$ \\ ${ }^{1}$ Department of Computer Science, King's College London, UK \\ joseph.chan@kcl.ac.uk \\ ${ }^{2}$ Department of Computer Science, The University of Hong Kong, Hong Kong \\ $\{$ chin, hfting\}@cs.hku.hk \\ ${ }^{3}$ Department of Computer Science and Engineering, Fudan University, China \\ 052021153@fudan.edu.cn
}

\begin{abstract}
A conflict-free coloring for a given set of disks is a coloring of the disks such that for any point $p$ on the plane there is a disk among the disks covering $p$ having a color different from that of the rest of the disks that covers $p$. In the dynamic offline setting, a sequence of disks is given, we have to color the disks one-by-one according to the order of the sequence and maintain the conflict-free property at any time for the disks that are colored. This paper focuses on unit disks, i.e., disks with radius one. We give an algorithm that colors a sequence of $n$ unit disks in the dynamic offline setting using $O(\log n)$ colors. The algorithm is asymptotically optimal because $\Omega(\log n)$ colors is necessary to color some set of $n$ unit disks for any value of $n$ [9.
\end{abstract}

\section{Introduction}

The conflict-free coloring (CF-coloring) problem was introduced by Even et al. 9] for modeling the frequency allocation problem that arises in wireless communication. In the frequency allocation problem, servers (base stations) and clients are connected by radio links. To establish a communication, a client scans the available frequencies in search for a base station with good reception. To avoid interference, the client needs to choose a specific frequency such that only one of the reachable base stations is assigned with that frequency. A naive solution is to assign each base station a distinct frequency. Since the spectrum is limited and costly, the target is to minimize the total number of frequencies assigned to the base stations such that for any client, among the frequencies assigned to all its reachable base stations, there is always a frequency assigned to exactly one of these stations.

In the CF-coloring problem, the geometric regions covered by the base stations are called ranges, and the goal is to find a coloring of these ranges (i.e., one color

\footnotetext{
* The research is partly supported by a Hong Kong RGC grant HKU7113/07E.

$\star \star$ The research is partly supported by a Hong Kong RGC grant HKU7171/08E. 
for each range) such that for every position that is covered by some subset of ranges, we can always find a range in the subset with a unique color. A formal and more general definition of CF-coloring is given as follows.

Definition 1 (Range Space). A Range Space is defined by a pair $(X, \mathcal{R})$ where $X$ is a set of vertices, and $\mathcal{R}$ is a family of subsets of $X$. The subsets in $\mathcal{R}$ are called ranges.

Definition 2 (CF-coloring). Let $(X, \mathcal{R})$ be a range space. A coloring $\chi: \mathcal{R} \rightarrow$ $N$, which maps $\mathcal{R}$ to the set of natural numbers $N$, is conflict-free if for every vertex $x \in X$, there is an $R \in \mathcal{R}$ such that $x \in R$ and $\chi(R) \neq \chi\left(R^{\prime}\right)$ for all other ranges $R^{\prime}$ containing $x$.

In the literature, the frequency allocation problem was also modeled as a dual of the CF-coloring problem [9]. The dual problem treats the base stations as vertices and ranges as the subsets of base stations within some geometric regions. The goal of the dual problem is to find a conflict-free coloring of the vertices such that for any range, there is always a vertex among the vertices in the range with a unique color.

Definition 3 (Dual CF-Coloring). Let $(X, \mathcal{R})$ be a range space. A coloring $\psi: X \rightarrow N$ is conflict-free if for every range $R \in \mathcal{R}$, there exists a vertex $x \in R$ such that $\psi(x) \neq \psi\left(x^{\prime}\right)$ for all other $x^{\prime} \in R$.

Note that the dual CF-coloring problem can be seen as a generalization of the traditional graph coloring problem. We can consider a range space as a hypergraph. When every range in the range space contains exactly two vertices, the range space is equivalent to a graph where a dual $\mathrm{CF}$-coloring of the range space is also a vertex coloring of the graph.

The problem of CF-colorings was first studied by Even et al. 9] and the $\mathrm{PhD}$ work of Smorodinsky 13 . One of the results in 9] was about CF-coloring for $n$ disks (of arbitrary size) on the plane. The range space $(X, \mathcal{R})$ of this problem is defined with $X$ equal to the set of all points on the plane and each range in $\mathcal{R}$ is a subset of $X$ covered by one of the $n$ disks. They showed that the range space, and equivalently the $n$ disks, can be colored with $O(\log n)$ colors. They also showed that $\Omega(\log n)$ colors are necessary even to color some set of $n$ unit disks for any value of $n$. Alon et al. 2] showed that if every disk intersects at most $k$ others, all disks can be colored with $O\left(\log ^{3} k\right)$ colors, no matter how many disks there are.

The dual CF-coloring problem with respect to disks was studied in $[9]$. The range space $(X, \mathcal{R})$ is defined by a given set $X$ of $n$ vertices on the plane. A range $R \subseteq X$ is in $\mathcal{R}$ if and only if there exists a disk that covers only the vertices in $R$. Even et al. 9] showed that a dual CF-coloring (on the $n$ vertices) can be constructed using $O(\log n)$ colors. A matching lower bound is proved by Pach and Tóth [12] that $\Omega(\log n)$ colors are required for CF-coloring every set of $n$ vertices on the plane with respect to disks.

The CF-coloring for rectangles and dual CF-coloring problems with respect to rectangles have also been studied in several recent papers $1,8,0,10,14$. 
The dual CF-coloring problem has been studied in the online model, where a sequence of vertices are presented one-by-one and each vertex is colored without knowledge of the vertices not yet presented. The assigned color of a vertex cannot be changed later and The coloring has to guarantee that at any time, the set of colored vertices holds the conflict-free property. Most of the previous works focused on coloring of $n$ vertices on a line where the ranges consist of all intervals of the vertices. It was shown in 9 that the static version of this problem can be solved using $1+\left\lfloor\log _{2} n\right\rfloor$ colors and this is also the best that we can do. For the online case, Chen et al. 6 gave an algorithm that uses $O\left(\log ^{2} n\right)$ colors. In between the static and the online models, Bar-Noy et al. 4 studied two semionline models, namely the dynamic offline model where the entire sequence of the vertices is given but the vertices have to be colored one-by-one according to the order of the sequence and the colors cannot be changed later, and the online absolute position model where the vertices are presented in the online fashion but the positions of all vertices on the line are known, i.e, for every vertex we know how many other vertices are on the left and right of this vertex. Bar-Noy et al. showed that $O(\log n)$ colors are sufficient to construct a dual CF-coloring in these two models.

Randomized algorithms have been proposed for the online dual CF-coloring for intervals [6], unit disks [5, 7], and hypergraphs (i.e., general range space) [3].

Our Contribution. This paper focuses on the dynamic offline version of the CFcoloring problem for unit disks (i.e., disks with radius one 1 . Let $\left\langle d_{1}, d_{2}, \ldots, d_{n}\right\rangle$ be a sequence of $n$ unit disks on the plane, which is given at the beginning. The required $\mathrm{CF}$-coloring $\chi$ of these $n$ disks satisfies the following requirement:

Property 1. For any $1 \leq i \leq n$, the coloring $\chi$ restricted to the subset of disks $D_{i}=\left\{d_{1}, d_{2}, \ldots, d_{i}\right\}$ is conflict-free, i.e., for any point $x$ covered by such disks, there is one $d \in D_{i}$ such that $d$ covers $x$ and for all other $d^{\prime} \in D_{i}$ that cover $x, \chi(d) \neq \chi\left(d^{\prime}\right)$.

To solve the above problem, we define and solve a dual CF-coloring problem with respect to unit disks in the dynamic offline setting that is to find a coloring $\psi$ for a sequence of $n$ vertices $\left\langle c_{1}, c_{2}, \ldots, c_{n}\right\rangle$ satisfying the following requirement.

Property 2. For any $1 \leq i \leq n$, the coloring $\psi$ restricted to the subset of vertices $C_{i}=\left\{c_{1}, c_{2}, \ldots, c_{i}\right\}$ is conflict-free, i.e., for any unit disk covering a nonempty subset of vertices of $C_{i}$, there is one $c \in C_{i}$ such that the unit disk covers $c$ and for all other $c^{\prime} \in C_{i}$ that is covered by the unit disk, $\psi(c) \neq \psi\left(c^{\prime}\right)$.

We show that the primal and dual problems defined above are equivalent in the sense that that they can be reduced to each other. The dual problem (in the dynamic offline setting) is then solved by constructing an equivalent but static dual CF-coloring problem. We show that the ranges of this static problem have a nice property so that we can find a dual CF-coloring with $O(\log n)$ colors.

\footnotetext{
${ }^{1}$ It is equivalent to to saying disks with fixed radius for the problem.
} 
This dual CF-coloring gives immediately, in the dynamic offline setting, a dual $\mathrm{CF}$-coloring for $n$ vertices where ranges are defined by units disks, as well as the corresponding primal problem for coloring unit disks, using $O(\log n)$ colors.

Our result is interesting theoretically. As mentioned above, the static version of the CF-coloring problem for $n$ disks requires $\Omega(\log n)$ colors even when the disks are restricted to unit disks. In fact, the conventional technique of solving the problems is by reducing the problem to the coloring problem on simple graphs. However, for unit disks, this technique might not work, as some range spaces can only be represented by hypergraphs with hyperedges having more than 2 elements. On the other hand, Even et al. 9] gave an example showing that for the dynamic offline case, $n$ colors are necessary for coloring $n$ disks with arbitrary radius. This paper shows that for the dynamic offline case coloring unit disks can be done much more efficiently than disks of arbitrary size, where $O(\log n)$ colors suffice.

Our algorithm has practical applications. First, base stations could have the same power and thus the areas covered by them are congruent disks. Second, the base stations are built according to some order, and before all of them are built, we still need a conflict-free frequency assignment to those stations that have been built.

The paper is organized as follows. In Section 2, we give a high-level algorithm that finds a dual CF-coloring for any given range space. We also show a modification of the algorithm, which becomes crucial in Section 4 in bounding the number of colors used. In Section 3, we show how the dynamic offline problem of CF-coloring unit disks can be transformed to an equivalent but static dual CF-coloring of range space. In Section 4, we first outline how the static problem obtained in Section 3 can be decomposed into sub-problems, and explain why we can focus on the sub-problems only. We proceed to show that the sub-problem can be solved using $O(\log n)$ colors, and hence the static dual CF-coloring problem defined in Section [3, as well as the one for unit disks can be solved using $O(\log n)$ colors.

\section{An Algorithm to Find a Dual CF-Coloring}

In this section, we describe a high-level algorithm of Even et al. 9 that uses the independent set approach for constructing a dual CF-coloring for any range space $(X, \mathcal{R})$. The following notation and definition are used in the algorithm. For any subset $S \subseteq X$ and any $1 \leq i \leq n$, define

$$
\mathcal{R} \|_{S}=\{R \cap S \mid R \in \mathcal{R}\}
$$

to be the set of ranges in $\mathcal{R}$ with all elements not in $S$ removed. For any $I \subseteq X$, we say that $I$ is an independent set of $(X, \mathcal{R})$ if for any range $R \in \mathcal{R}$ with $|R| \geq 2$, we have $R \nsubseteq I$.

Algorithm $\mathrm{CF}$-color constructs a dual $\mathrm{CF}$-coloring for $(X, \mathcal{R})$ by assigning the same color to the vertices in an independent set of $(X, \mathcal{R})$ and repeat the process using new colors for the range space induced by the uncolored vertices 
until all vertices are colored. It is not difficult to see that the resulting coloring is conflict-free.

Theorem 1 (Even et al. [9]). The coloring $\chi$ of $X$ constructed by Algorithm $\mathrm{CF}$-Color is a dual CF-coloring on $(X, \mathcal{R})$.

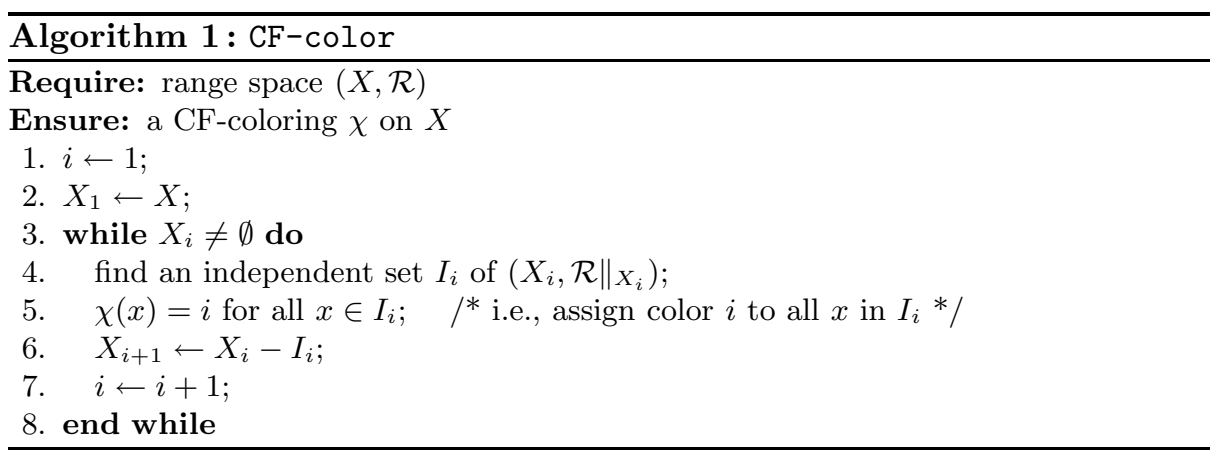

To bound the number of colors used to $O(\log n)$, it suffices to make sure that in each iteration the size of the independent set is a constant fraction of the number of vertices in the range space, so that $O(\log n)$ iterations, as well as colors, are sufficient. The rest of the paper is devoted to applying this idea to the problem of CF-coloring for unit disks in the dynamic offline setting.

\subsection{Modified Algorithm Using Min-Range Subset}

Define the min-range subset of a set $\mathcal{R}$ of ranges to be

$$
\min (\mathcal{R})=\left\{R \in \mathcal{R}|| R \mid \geq 2 \text { and } \nexists R^{\prime} \in \mathcal{R} \text { such that }\left|R^{\prime}\right|>2 \text { and } R^{\prime} \varsubsetneqq R\right\},
$$

i.e., the set of non-trivial ranges in $\mathcal{R}$ that is minimum by set inclusion. The following lemma makes sure that to find an independent set of a range space we can focus on the min-range subset.

Lemma 1. Given a range space $(X, \mathcal{R})$, an independent set of the range space $(X, \min (\mathcal{R}))$ is also an independent set of $(X, \mathcal{R})$.

Proof. Consider an independent set $I$ for $(X, \min (\mathcal{R}))$. Suppose to the contrary that $I$ is not independent for $(X, \mathcal{R})$. There is a range $R \in \mathcal{R}$ with $|R| \geq 2$ such that $R \subseteq I$. Since $\min (\mathcal{R})$ is a min-range subset of $\mathcal{R}$, we have either $R \in \min (\mathcal{R})$ or there is another range $R^{\prime} \in \min (\mathcal{R})$ with $R^{\prime} \subset R$. In both cases, there is a range in $\min (\mathcal{R}), R$ or $R^{\prime}$, which is of size at least 2 and is a subset of or equal to $I$. It contradicts to the assumption that $I$ is an independent set of $(X, \min (\mathcal{R}))$.

We modify the Algorithm CF-Color in Step 4 as follows.

If $\min \left(\mathcal{R} \|_{X_{i}}\right) \neq \emptyset$, find an independent set $I_{i}$ of $\left(X_{i}, \min \left(\mathcal{R} \|_{X_{i}}\right)\right)$; else $I_{i} \leftarrow X_{i}$. 
There is a case where every range in $\mathcal{R} \|_{X_{i}}$ is a subset of $X_{i}$ with single element. We can take $X_{i}$ as the independent set. By Lemma 1, the modified algorithm works as the original one. The reason that we use min-range subset will become clear in Section 4.1 when we show that the independent set thus obtained consists of at least a constant fraction of vertices of $X_{i}$. This helps to bound the number of colors used in the coloring.

\section{Dynamic Offline CF-Coloring for Unit Disks}

In this section, we show that the dynamic offline CF-coloring problem for $n$ unit disks can be transformed to a static dual CF-coloring problem on some special range space. Then, in the rest of the paper, we show how to solve this dual problem using $O(\log n)$ colors.

We first explain how to transform the static CF-coloring problem for $n$ unit disks to its dual problem as follows. Let $D=\left\{d_{1}, d_{2}, \ldots, d_{n}\right\}$ be a set of $n$ unit disks. For each $1 \leq i \leq n$, let $c_{i}$ be the center of the unit disk $d_{i}$. Define the range space $(C, \mathcal{R})$ where $C=\left\{c_{1}, c_{2}, \ldots, c_{n}\right\}$ and

$$
\mathcal{R}=\{R \subseteq C \mid \exists \text { a unit disk (not necessarily from } D \text { ) that covers exactly } R\} .
$$

A more general form of the following lemma has been shown in [9] that the static $\mathrm{CF}$-coloring for $D$ can be reduced to the static dual $\mathrm{CF}$-coloring on $(C, \mathcal{R})$.

Lemma 2 (Even et al. [9]). Consider a set of unit disks $D$ and the range space $(C, \mathcal{R})$ defined above. Let $\psi: C \rightarrow N$ be a dual $C F$-coloring on $(C, \mathcal{R})$. Then, the coloring $\chi: D \rightarrow N$ where $\chi\left(d_{i}\right)=\psi\left(c_{i}\right)$ for $1 \leq i \leq n$ is a CF-coloring for $D$.

The following lemma extends Lemma 2 to the dynamic offline setting.

Lemma 3. Consider a sequence of unit disks $D$ and the corresponding sequence of centers $C$. Let $\psi: C \rightarrow N$ be a dual CF-coloring of $C$ satisfying the dynamic offline Property 2 (in the Introduction). Then, the coloring $\chi: D \rightarrow N$ where $\chi\left(d_{i}\right)=\psi\left(c_{i}\right)$ for $1 \leq i \leq n$ is a CF-coloring for $D$ satisfying the dynamic offline Property 1 (in the Introduction).

Proof. For any $1 \leq i \leq n$, let $C_{i}=\left\{c_{1}, c_{2}, \ldots, c_{i}\right\}$ and $\mathcal{R}_{C_{i}}=\left\{R \subseteq C_{i} \mid \exists\right.$ a unit disk covers $R$ but no other centers in $C_{i}$. The lemma is true because according to Property $2, \psi$ is a dual CF-coloring of $\left(C_{i}, \mathcal{R}_{C_{i}}\right)$ for any $1 \leq i \leq n$. Then, by Lemma 2, $\chi$ is a CF-coloring for $D_{i}$ for any $1 \leq i \leq n$, and hence $\chi$ is a CF-coloring for $D$ satisfying Property 1.

To reduce the problem from the dynamic offline setting to a static setting, we need some definition. For any $1 \leq i \leq n$, let $C_{i}=\left\{c_{1}, c_{2}, \ldots, c_{i}\right\}$ and define

$\mathcal{R}_{C_{i}}=\left\{R \subseteq C_{i} \mid \exists\right.$ a unit disk covers $R$ but no other centers in $\left.C_{i}\right\}$,

and $\mathcal{R}_{C}=\bigcup_{1 \leq i \leq n} \mathcal{R}_{C_{i}}$. The following lemma is suggested by Bar-Noy et al. [4] (in a more general form) to reduce problem from the dynamic offline setting to the static setting. 
Lemma 4 (Bar-Noy et al. [4]). Consider a sequence of centers $C$ and the range space $\left(C, \mathcal{R}_{\mathcal{C}}\right)$ defined above. Let $\psi: C \rightarrow N$ be a dual $C F$-coloring on $\left(C, \mathcal{R}_{C}\right)$. Then $\psi$ is also a dual CF-coloring for $C$ that satisfies the dynamic offline Property 2.

\section{Dual CF-Coloring of $\left(C, \mathcal{R}_{C}\right)$ with $O(\log n)$ Colors}

In this section, we show how to find a dual CF-coloring of $\left(C, \mathcal{R}_{C}\right)$ defined by a sequence of unit disks in Section 3 using the modified Algorithm CF-Color of Section 2 with $O(\log n)$ colors. There are mainly two ideas that lead us to the bound.

1. The plane can be partitioned into unit hexagons (with each side of length one). Note that the hexagons can be divided into seven groups such that no two hexagons in the same group intersect with the same unit disk. See Figure 1. The set of centers $C$ is partitioned into disjoint subsets such that each subset consists of centers of $C$ within the same unit hexagon. For each subset $F$ thus defined, we have a range space $\left(F, \mathcal{R}_{C} \|_{F}\right)$ which we can color independently, provided that we are using seven sets of different colors where one set for each group of hexagons and the corresponding subsets of centers.

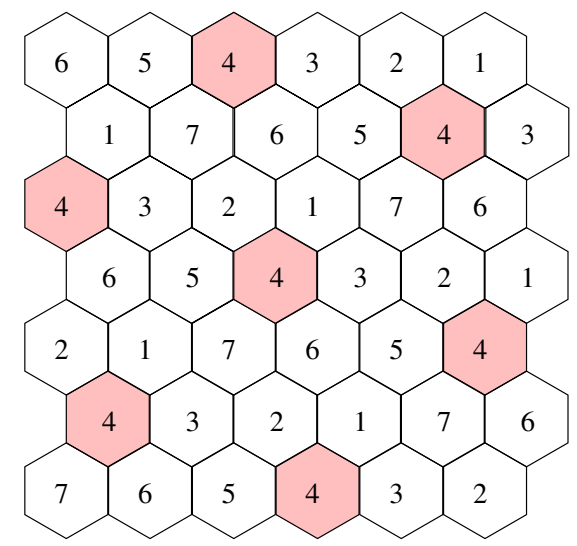

Fig. 1. The plane is partitioned into hexagons

2. We show (in Section 4.1) that the dual CF-coloring of $\left(F, \mathcal{R}_{C} \|_{F}\right)$ can be obtained by using the modified Algorithm CF-Color with $O(\log n)$ colors, where $F \subseteq C$ is a subset of centers within the same unit hexagon.

Combining the two ideas, we can find the dual CF-coloring for $\left(C, \mathcal{R}_{C}\right)$ with $O(\log n)$ colors, as well as a dynamic offline CF-coloring for a sequence of $n$ unit disks. The theorem below follows.

Theorem 2. We can construct a dynamic offline CF-coloring for a sequence of $n$ unit disks using $O(\log n)$ colors. 


\subsection{Dual CF-Coloring in a Unit Hexagon}

In this section, we assume that the centers in $C=\left\{c_{1}, c_{2}, \ldots, c_{n}\right\}$ are all within a unit hexagon. We show how $\left(C, \mathcal{R}_{C}\right)$ can be colored by the modified Algorithm $\mathrm{CF}$-Color using $O(\log n)$ colors. The input for the algorithm $X$ and $\mathcal{R}$, are $C$ and $\mathcal{R}_{C}$, respectively. Recall that in each iteration of the algorithm, say the $i$-th iteration, we need to find an independent set $I_{i}$ of $\left(X_{i}, \min \left(\mathcal{R} \|_{X_{i}}\right)\right)$ and remove the centers in $I_{i}$ from $X_{i}$ before we continue in the next iteration. The key property for us to prove the $O(\log n)$ bound is that $I_{i}$ consists of a constant fraction of $X_{i}$. Therefore, the number of iteration, as well as the number of colors used, is at most $O\left(\log \left|X_{1}\right|\right)$, i.e., $O(\log |C|)$ or $O(\log n)$. To prove the key property, we prove the following claims.

Given any subset $Y \subseteq C$, let $G$ be the range space $\left(Y, \min \left(\mathcal{R}_{C} \|_{Y}\right)\right)$.

1. $G$ is indeed a simple graph, i.e., each of its ranges consists of two centers (Lemma 51), and

2. $G$ consists of $O(n)$ edges (Lemma 91).

With the second claims, we can guarantee that $G$ has an independent set of size $\Omega(n)$ and an independent set of size $\Omega(n)$ can be found in polynomial time [1]. Thus, the following theorem follows.

Theorem 3. We can construct a dynamic offline dual CF-coloring with respect to unit disks for a sequence of $n$ vertices (or points) all within a unit hexagon using $O(\log n)$ colors.

The remainder of the section focuses on proving Lemmas 5 and 9] First, we show that for any $Y \subseteq C,\left(Y, \min \left(\mathcal{R}_{C} \|_{Y}\right)\right)$ corresponds to a simple graph 2 .

Lemma 5. For any $Y \subseteq C$, all $R \in \min \left(\mathcal{R}_{C} \|_{Y}\right)$ have $|R|=2$.

Proof. Suppose that $R=\left\{c_{i_{1}}, c_{i_{2}}, \ldots, c_{i_{k}}\right\} \in \min \left(\mathcal{R}_{C} \|_{Y}\right)$ where $i_{1}<i_{2}<\cdots<$ $i_{k}$. We have $|R|=k$. There is a unit disk $d$ that covers all centers in $R$ but no other centers $c_{j}$ for $j \leq i_{k}$. It follows that $d$ also covers only $c_{i_{1}}$ and $c_{i_{2}}$ at the stage when center $c_{i_{2}}$ is presented. Therefore, we have $\left\{c_{i_{1}}, c_{i_{2}}\right\} \in \min \left(\mathcal{R}_{C} \|_{Y}\right)$. As the ranges are min-ranges, $k$ must be 2 ; otherwise $\left\{c_{i_{1}}, c_{i_{2}}\right\}$ is a proper subset of $R$, which is a contradiction.

To analyze the graph structure of $G$, which corresponds to $\left(C, \min \left(\mathcal{R}_{C}\right)\right.$ ) (or equivalently $\left(Y, \min \left(\mathcal{R}_{C} \|_{Y}\right)\right)$ as $Y$ can be $\left.C\right)$, we consider the geometric property of the unit disks $D=\left\{d_{1}, d_{2}, \ldots, d_{n}\right\}$ where $d_{i}$ is centered at $c_{i}$. Let $A_{D}$ denote the partition of the plane by the unit disks in $D$. A face is defined to be a partition in $A_{D}$, i.e., a face is the maximal contiguous region covered by the same set of unit disks in $D . A_{D_{i}}$ is defined similarly as $A_{D}$ but it corresponds to the partition by $D_{i}=\left\{d_{1}, d_{2} \ldots, d_{i}\right\}$. See Figure 4 for an example. We say that a face is lv- $k$ if the face is covered by $k$ unit disks.

${ }^{2}$ For the static dual CF-coloring (of a set of points) with respect to disks [9], this simple graph is in fact the Delaunay graph induced by the set of points. However, in the dynamic offline case, the graph may not be the Delaunay graph. 
We first give two properties, Lemmas 6 and 7 of $A_{D}$ with respect to a particular unit disk.

Lemma 6. Let $D$ be a set of unit disks whose centers are within a unit hexagon. For any $d \in D$, there are at most two lv-1 faces in $A_{D}$ covered by $d$.

Proof. For any three disks whose centers are in a unit hexagon, they must all intersect at least one point (or a face). Figure 2 and 3 show the two different cases. For Case 1 , let $p_{1}, p_{2}$ and $p_{3}$ be the three intersection points at the outer boundary of the union of the three disks. It can be proved that any unit disk is too small to inscribe all the three points $p_{1}, p_{2}$ and $p_{3}$ so as to form three lv-1 faces of a unit disk. For Case 2, again there is no way for the new unit disk to create three lv-1 faces.

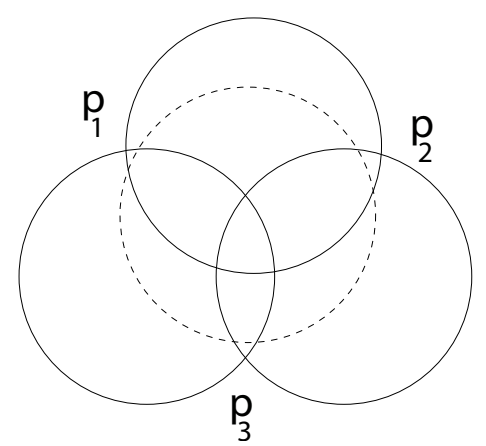

Fig. 2. Three unit disks intersect with each other: Case 1

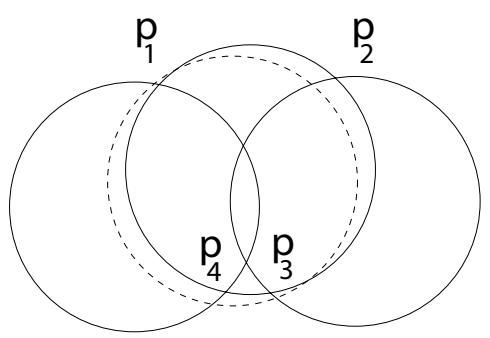

Fig. 3. Three unit disks intersect with each other: Case 2

The following lemma give a bound of the number of a special kind of lv-2 faces of a unit disk, which is defined as follows. For a unit disk $d \in D$, a $p$-lv-2 faces of $d$ in $A_{D}$ is defined to be a maximal intersecting region of $d$ and a lv-1 face in $A_{D-\{d\}}$ and the intersecting region is strictly smaller than the lv-1 face. (Note that p-lv-2 faces are subjected to unit disks. For example in Figure 4, $f_{1}$ is a lv-2 face of both $d_{1}$ and $d_{2}$ but $f_{1}$ is a p-lv-2 face of $d_{2}$ but not a p-lv-2 face of $d_{1}$.)

Lemma 7. Let $D$ be a set of unit disks whose centers are within a unit hexagon. For any $d \in D, d$ has at most nine $p$-lv-2 faces in $A_{D}$, each of the faces corresponds to $d$ intersecting a distinct unit disk in $D$.

Proof. We prove by contradiction. Assume that $d$ has ten or more p-lv-2 faces. For each p-lv-2 face, it shares part of the boundary with $d$ because by the definition of $\mathrm{p}-\mathrm{lv}-2$ face the face must be next to some $1 \mathrm{v}-1$ face of a disk other than $d$. For each of the ten p-lv-2 faces, select a point in the face which is on the boundary of $d$, denoted as f-point. Consider five of the f-points in alternate positions. See Figure 5. Out of the five f-points, there are at least three of them with the property. 


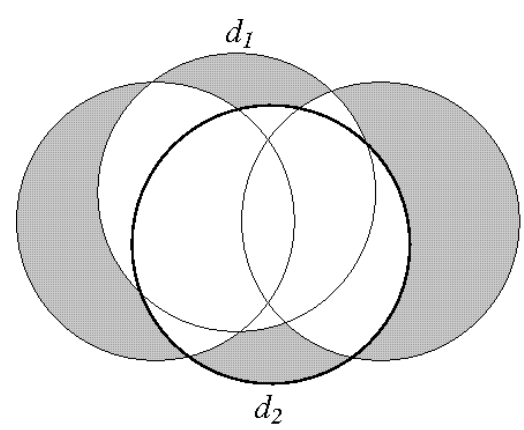

lv-1 faces

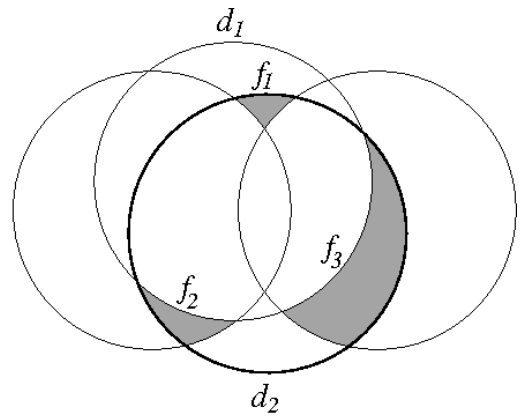

lv-2 faces

Fig. 4. An example of $l v-1$ and $l v-2$ faces

For an f-point $p$, the part of the boundary of $d$ defined by the f-points on its left $p_{\ell}$ and right $p_{r}$ occupies at most $1 / 3$ of the whole circumference of $d$. Effectively, every point on the boundary of $d$ from $p_{\ell}$ to $p_{r}$ through $p$ has a distance at most 1 from either $p_{\ell}$ or $p_{r}$.

Let $p_{1}, p_{2}$ and $p_{3}$ be the three f-points satisfying the property. Let $c_{1}, c_{2}$ and $c_{3}$ be the centers of three disks in $D$ that intersect $d$ to form the p-lv-2 faces defined by $p_{1}, p_{2}$ and $p_{3}$, respectively. By the property, all $c_{1}, c_{2}$ and $c_{3}$ must be outside $d$ (because the distance between $p_{\ell}$ and $p_{r}$ is short) and all f-points are outside the disks centered at $c_{1}, c_{2}$ and $c_{3}$, except $p_{1}, p_{2}$ and $p_{3}$, respectively. See Figure 5. It follows that there is no region on the plane covered by $d$ and all disks centered at $c_{1}, c_{2}, c_{3}$. In other words, $c_{1}, c_{2}, c_{3}$ and the center of $d$, are not in the same unit disk or unit hexagon, which is a contradiction to the assumption.

Recall that our task is to bound the number of edges in $G$, where $G$ is the simple graph corresponding to $\left(C, \min \left(\mathcal{R}_{C}\right)\right)$. The following lemma corresponds the edges in $G$ with the lv-2 faces in $A_{D_{j}}$ for some $j$.

Lemma 8. There is an edge $\left(c_{i}, c_{j}\right)$ in $G$ for $i<j$ if and only if there is a lv-2 face covered by unit disks $d_{i}$ and $d_{j}$ in $A_{D_{j}}$.

Proof. If $\left(c_{i}, c_{j}\right)$ is in $G$, then $\left\{c_{i}, c_{j}\right\} \in \min \left(\mathcal{R}_{C_{j}}\right) \subseteq \mathcal{R}_{C_{j}}$. There is a unit disk $d$ covering $c_{i}$ and $c_{j}$ but no other $c_{k}$ for $k \leq j$. It follows that the center of $d$ is at a distance more than one with the other $c_{k}$. Thus there is some region covered only by $c_{i}$ and $c_{j}$ in $A_{D_{j}}$, which is a lv-2 face. Similarly, we can prove the other way round.

We are now ready to bound the number of edges in $G$.

Lemma 9. There are at most $11 n-1$ edges in $G$ where $n$ is the number of centers in $C$.

Proof. For $1 \leq k \leq n$, let $\operatorname{deg}^{-}\left(c_{k}\right)$ denote the number of edges $\left(c_{k}, c_{i}\right)$ in $G$ for $i<k$. By Lemma 8 , $\operatorname{deg}^{-}\left(c_{k}\right)$ is equal to the number of lv-2 faces of $d_{k}$ 

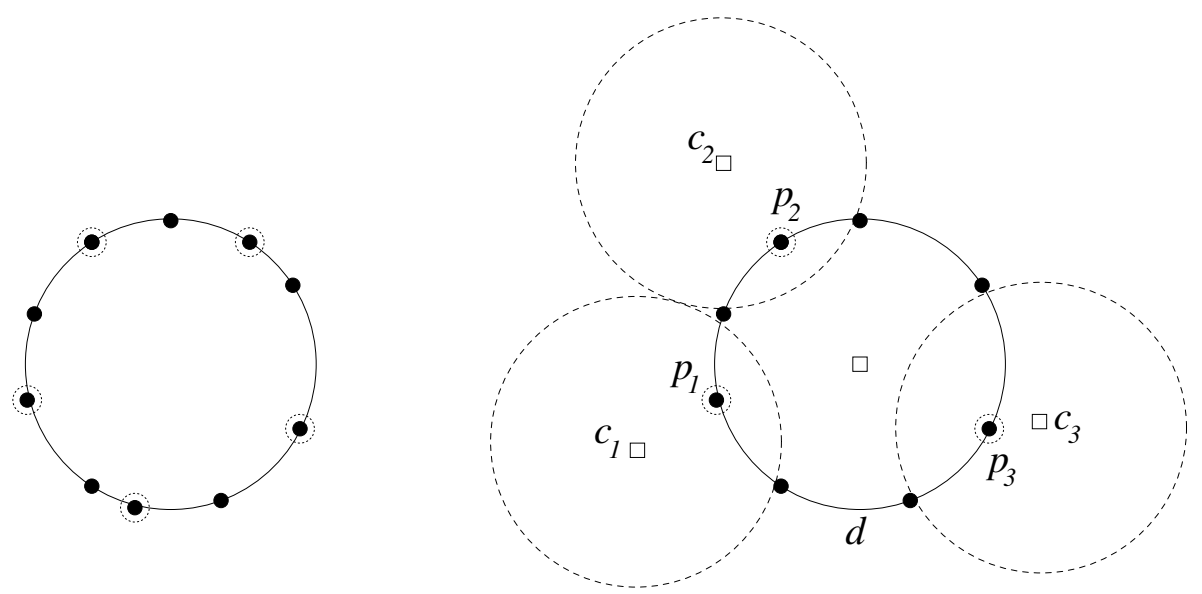

Fig. 5. Left: Ten f-points on the boundary of a disk, five highlighted in alternate positions. Right: The three chosen f-points $p_{1}, p_{2}$ and $p_{3}$ and the corresponding disks centered at $c_{1}, c_{2}$ and $c_{3}$ that cover them.

in $A_{D_{k}}$. Let pcover $(k)$ be the number of p-lv-2 faces of $d_{k}$ in $A_{D_{k}}$. Define a $f-l v-2$ face of $d_{k}$ to be a lv-2 face but not a p-lv-2 face of $d_{k}$. Let $f \operatorname{cover}(k)=$ $\operatorname{deg}^{-}\left(c_{k}\right)-\operatorname{pcover}(k)$ denote the number of $\mathrm{f}-\mathrm{lv}-2$ faces of $d_{k}$. Let create $(k)$ be the number of lv-1 faces of $d_{k}$ in $A_{D_{k}}$. Since each f-lv-2 face of $d_{k}$ must correspond to a distinct lv- 1 face of some $d_{i}$ in $A_{D_{k-1}}$, i.e., $i<k$, overall, we have $\sum_{1 \leq k \leq n}$ create $(i) \geq \sum_{1 \leq k \leq n}$ fcover $(i)$.

Together with Lemma 7 the number of edges in $G$ is equal to

$$
\begin{aligned}
\sum_{1 \leq k \leq n} \operatorname{deg}^{-}\left(c_{k}\right) & =\sum_{1 \leq k \leq n}(\operatorname{pcover}(k)+\mathrm{f} \operatorname{cover}(k)) \\
& \leq \sum_{1 \leq k \leq n}(9+\mathrm{f} \operatorname{cover}(k)) \\
& \leq 9 n+\sum_{1 \leq k \leq n} \operatorname{create}(k) \\
& \left.\leq 9 n+2 n-1 \quad \text { since } d_{1} \text { has only one lv-1 face in } A_{D_{1}}\right\} \\
& \leq 11 n-1
\end{aligned}
$$

\section{Remarks and Open Problems}

Although the paper focuses on minimizing the colors used in constructing the $\mathrm{CF}$-coloring, we would like to mention the time complexity of our approach. By the Algorithm $\mathrm{CF}$-color, the running time mainly depends on the time to find an independent set in a simple graph times the number of iterations, which turns out to be $O(\log n)$ if the size of the independent set is a constant fraction of that of the vertex set. Hochbaum 11] gave an algorithm that finds an independent set (of a graph having linear number of edges) with size $\Omega(n)$ in $O\left(n^{3 / 2}\right)$ time. As a result, our algorithm runs in $O\left(n^{3 / 2} \log n\right)$ time. 
In the dynamic offline model, we are given the whole sequence of disks at the beginning, so that the corresponding range space is known and fixed. That is why the general framework of the independent set method works. However, in the online (or even the online absolute position) settings, the range space is not known in advance and thus the general framework cannot apply. An interesting open problem is to develop a deterministic algorithm to solve the online version of the problem with polylog number of colors.

\section{References}

1. Ajwani, D., Elbassioni, K.M., Govindarajan, S., Ray, S.: Conflict-free coloring for rectangle ranges using $O\left(n^{.382}\right)$ colors. In: The 19th Annual ACM Symposium on Parallelism in Algorithms and Architectures (SPAA), pp. 181-187 (2007)

2. Alon, N., Smorodinsky, S.: Conflict-free colorings of shallow discs. In: The 22nd ACM Symposium on Computational Geometry (SoCG), pp. 41-43 (2006)

3. Bar-Noy, A., Cheilaris, P., Olonetsky, S., Smorodinsky, S.: Online conflict-free colorings for hypergraphs. In: Arge, L., Cachin, C., Jurdziński, T., Tarlecki, A. (eds.) ICALP 2007. LNCS, vol. 4596, pp. 219-230. Springer, Heidelberg (2007)

4. Bar-Noy, A., Cheilaris, P., Smorodinsky, S.: Conflict-free coloring for intervals: from offline to online. In: The 18th Annual ACM Symposium on Parallel Algorithms and Architectures (SPAA), pp. 128-137 (2006)

5. Chen, K.: How to play a coloring game against a color-blind adversary. In: The 22nd ACM Symposium on Computational Geometry (SoCG), pp. 44-51 (2006)

6. Chen, K., Fiat, A., Kaplan, H., Levy, M., Matousek, J., Mossel, E., Pach, J., Sharir, M., Smorodinsky, S., Wagner, U., Welzl, E.: Online conflict-free coloring for intervals. SIAM J. Comput. 36(5), 1342-1359 (2007)

7. Chen, K., Kaplan, H., Sharir, M.: Online conflict-free coloring for halfplanes, congruent disks, and axis-parallel rectangles. ACM Transactions on Algorithms (in press)

8. Elbassioni, K.M., Mustafa, N.H.: Conflict-free colorings of rectangles ranges. In: Durand, B., Thomas, W. (eds.) STACS 2006. LNCS, vol. 3884, pp. 254-263. Springer, Heidelberg (2006)

9. Even, G., Lotker, Z., Ron, D., Smorodinsky, S.: Conflict-free colorings of simple geometric regions with applications to frequency assignment in cellular networks. SIAM J. Comput. 33(1), 94-136 (2003)

10. Har-Peled, S., Smorodinsky, S.: Conflict-free coloring of points and simple regions in the plane. Discrete \& Computational Geometry 34(1), 47-70 (2005)

11. Hochbaum, D.S.: Efficient bounds for the stable set, vertex cover and set packing problems. Discrete Applied Mathematics 6(3), 243-254 (1983)

12. Pach, J., Tóth, G.: Conflict-free colorings. In: Aronov, B., Basu, S., Pach, J., Sharir, M. (eds.) Discrete and Computational Geometry - The Goodman-Pollack Festschrift. Springer, Heidelberg (2003)

13. Smorodinsky, S.: Combinatorial Problems in Computational Geometry. PhD thesis, Tel-Aviv University (2003)

14. Smorodinsky, S.: On the chromatic number of some geometric hypergraphs. In: The 17th Annual ACM-SIAM Symposium on Discrete Algorithms (SODA), pp. 316-323 (2006) 